\title{
EDITORIAL
}

\section{El creciente desafío del proceso editorial en la Revista Médica de Chile}

\author{
The growing challenge of \\ the editorial process in \\ Revista Médica de Chile
}

During the recent 6 years, a growing number of manuscripts have been submitted annually to Revista Médica de Chile. In 2005 this number was $60 \%$ greater than in the year 2000. This determined an increased workload in the editorial handling of manuscripts and in the number of external peer reviewers, who were over 400 participants during 2004, each one reviewing from one to five manuscripts in that year. An increasing use of the electronic mail helps to communicate editors with reviewers and authors. However, there is yet no availability of a software in Spanish to handle all communications in the editorial process in this language. The number of pages per issue has been increased and authors have been requested to limit the extension of their manuscripts, in order to allow more accepted manuscripts to appear in a shorter period of time. At the present, an average of 8.9 months separates the date of submission to the date of publication, in those manuscripts that were accepted in revised version after external peer review. The increasing number of submissions and the fact that this journal ranks high among Chilean journals, in the number of visits to full-text articles in the web page www.scielo.cl, testify that in spite of the aforementioned limitations Revista Médica de Chile is well considered among authors and readers in Chile and other Spanish-speaking countries. (Rev Méd Chile 2006; 134: 7-11)

(Key-w ords: Journalism, medical; Manuscripts, medical; Peer review; Periodicals)

$\mathrm{E}^{1}$ comienzo de un nuevo año es oportunidad propicia para que los editores revisemos el estado actual del proceso editorial, enfocándolo en nuestras relaciones con los lectores (a quienes está destinada la Revista), con los autores que solicitan espacio para sus manuscritos, y con otras revistas del país y del extranjero. Este análisis puede ser útil para apreciar la magnitud de eventuales problemas y diseñar una estrategia para resolverlos.

Las relaciones de la Revista con sus lectores son difíciles de evaluar porque carecemos de índices numéricos adecuados: ¿Cuántos lectores tiene la Revista?; ¿Cuántas personas la consultan en las bibliotecas?; ¿Quiénes son sus lectores? (¿médicos?, ¿otros profesionales?, ¿especialistas o generalistas?, ¿estudiantes?); ¿Cuáles fueron los artículos preferidos por ellos?; ¿Qué trascendencia tiene la información que reciben?; ¿Qué proporción de los contenidos de la Revista se incorporaron a la cultura profesional de sus lectores gracias al estímulo de haberlos leído aquí?

Una medida de uso común para apreciar la popularidad de una revista es el número de suscriptores, pero merece algunas consideraciones: 1. La condición de suscriptor se adquiere muchas veces como parte de los beneficios (y no el único) de ser miembro de la entidad que edita la Revista y tal es el caso de la mayoría de nuestros suscriptores; 2 . Otra vía de acceso ofrecen las bibliotecas médicas a las que llega la Revista impresa, quedando a disposición de sus 
consultantes, entre quienes se cuentan los estudiantes de medicina y otras carreras de la salud; 3 . El texto completo de los artículos es accesible gratuitamente en una versión electrónica, como ocurre con la Revista Médica de Chile en el sitio web www.scielo.cl.

Nuestra Revista fue una de las primeras que se incorporó al programa SciELO Chile, de la Comisión Nacional de Investigación Científica y Tecnológica (CONICYT) ${ }^{1}$. Por convenio con la Sociedad Médica de Santiago, ambas instituciones tomaron medidas para publicar una versión electrónica de la Revista. Sin embargo, ésta aparece con cierto retraso en relación con la versión en papel y muchos autores de manuscritos consideran indispensable la publicación en formato impreso.

CONICYT dispone de un programa computacional que permite conocer, entre otros datos, el número de veces que los lectores han accedido al texto completo de cada manuscrito en las revistas indizadas en SciELO Chile. La Tabla 1 muestra el número de visitas que recibieron siete revistas del área de salud en los cuatro años recientes, resaltando la Revista Médica de Chile y la Revista Chilena de Pediatría, con cerca de 500.000 visitas anuales a cada una. Lamentablemente este programa no permite averiguar el lugar desde donde se
Tabla 1. Visitas a revistas clínicas en el sitio web de SCiE LO -C hile (octubre 2001 a octubre de 2005)

\begin{tabular}{|lc|}
\hline Revista & № visitas a artículos \\
\hline Rev Méd Chile & 1.866 .253 \\
Rev Chil Pediat & 1.366 .731 \\
Rev Chil Obstet Ginecol & 814.151 \\
Rev Chil Infectol & 631.082 \\
Parasitol Latinoam & 366.939 \\
Rev Chil Enf Resp & 210.347 \\
Bol Chil Parasitol & 114.027 \\
\hline
\end{tabular}

consultaron las Revistas pero, dado que ambas se publican total o mayoritariamente en idioma español, podemos suponer que quienes accedieron al texto completo de los artículos proceden de países hispano parlantes. En cambio, los resúmenes en inglés están disponibles simultáneamente en los sitios web de SciELO Chile y de la National Library of Medicine, U.S.A. (PubMed).

El programa computacional aludido permite también verificar el número de veces que fue solicitado cada artículo. La Tabla 2 identifica los seis artículos más visitados en los últimos cuatro

\section{Tabla 2. La Revista Médica de Chile en el sitio web de SciE LO -C hile. Artículos más visitados (octubre 2001 a octubre 2005)}

\begin{tabular}{|c|c|}
\hline № Visitas & Artículo y fecha de publicación \\
\hline 9040 & $\begin{array}{l}\text { VizCARRA MB, CoRTes J, Bustos L ET AL. } \\
\text { Maltrato Infantil en la ciudad de Temuco. Estudio de prevalencia y factores asociados. } \\
\text { Diciembre } 2001 \text {. }\end{array}$ \\
\hline 8563 & $\begin{array}{l}\text { PAZ F, CÉSPEDES P, CUEvAS M ET AL. } \\
\text { Derrame pleural y empiema complicado en niños. Evolución y factores pronósticos. } \\
\text { Noviembre } 2001 \text {. }\end{array}$ \\
\hline 8432 & $\begin{array}{l}\text { MOLINA M, FERRADA C, PÉREZ R ET AL. } \\
\text { Embarazo en la adolescencia y su relación con la deserción escolar. Enero } 2004 .\end{array}$ \\
\hline 7683 & $\begin{array}{l}\text { SAPUNAR J, VIDAL T, BAUER K. } \\
\text { Anomalías de la esteroidogénesis suprarrenal en niños chilenos con micropene. Enero } 2003 .\end{array}$ \\
\hline 7609 & $\begin{array}{l}\text { CAIOZZI G, CABRERA D, MARDONES JM ET AL. } \\
\text { Hierbas medicinales y graves efectos adversos: Angioedema de úvula causado por el uso } \\
\text { de Ecballium olaterium (caso clínico). Diciembre } 2002 \text {. }\end{array}$ \\
\hline 7549 & $\begin{array}{l}\text { VALDÉS G, RoESSLER E. } \\
\text { Recomendaciones para el manejo de las crisis hipertensivas: Documento de consenso de } \\
\text { la Sociedad Chilena de Hipertensión Arterial. Marzo } 2002 \text {. }\end{array}$ \\
\hline
\end{tabular}


Tabla 3. Flujo de manuscritos en el Primer Semestre de 2000, 2003 y 2005

\begin{tabular}{|lrrr|}
\hline Manuscritos & 2000 & 2003 & 2005 \\
& $n$ & $n$ & $n$ \\
\hline Recibidos & 114 & 148 & 183 \\
Aceptados para publicación & 85 & 90 & 82 \\
Devueltos a autores & 14 & 40 & 52 \\
Rechazados & 15 & 18 & 36 \\
\hline
\end{tabular}

Tabla 4. Lapso de tiempo desde la recepción de un artículo hasta su publicación en la Revista Médica de Chile

\begin{tabular}{|lccc|}
\hline & 2000 & 2003 & 2005 \\
& Enero a Diciembre & Enero a Junio & Enero a Junio \\
\hline Artículos publicados & 99 & 52 & 86 \\
Lapso promedio (meses) & 7,5 & 7,6 & 8,9 \\
Rango (meses) & 4 a 13 & 3 a 12 & 3 a 15 \\
\hline
\end{tabular}

años, en la Revista Médica de Chile. Esta lista permitiría deducir que sus contenidos interesan a un público muy amplio, que sobrepasa el ámbito de la medicina interna y sus especialidades derivadas. Sin embargo, desconocemos el perfil profesional»de quienes consultan la versión electrónica (profesión, años de título, especialidad, lugar geográfico de residencia, etc.) y no es posible compararlos con aquellos que leen su versión impresa.

En relación con los autores de manuscritos, cabe destacar que se ha acentuado un fenómeno al que ya habíamos llamado la atención: el número de manuscritos recibidos crece progresivamente $^{2-4}$. En 2005 superó en $60 \%$ al período similar en el año 2000 (Tabla 3) y duplicó a los recibidos en años de la década precedente. Para afrontar esta situación, la secretaría de la Revista ha sido reforzada y se ha modemizado la tecnología disponible, pero la carga de trabajo es muy superior a la de hace diez años y sigue creciendo. Se ha modificado el manejo editorial de los manuscritos y se han acelerado las relaciones con los autores y los revisores externos mediante el correo electrónico, pero diversos factores hacen necesario mantener parcialmente el uso del correo tradicional y de mensajero (estafeta) para la correspondencia confidencial con los revisores externos. Todo ello influye en el tiempo que media desde la recepción de un manuscrito hasta que los editores deciden aceptarlo o rechazarlo, y luego desde la fecha de aceptación hasta que el manuscrito final tiene cabida en un número de la Revista, cuyo cupo de páginas es limitado. Las etapas de este proceso fueron analizadas en una Editorial previa ${ }^{2}$. En 2005, el lapso promedio desde la recepción de un manuscrito hasta su publicación fue de 8,9 meses, solo levemente mayor que en años previos, lo que refleja el esfuerzo realizado para afrontar la mayor carga de manuscritos (Tabla 4). Sin embargo, numerosos autores han expresado su disconformidad por esta demora (no absorbieron la alusión al poema (T.T.T.») y temen que lo que escribieron 3 a 4 meses antes (su versión corregida después de la revisión por pares y por los editores) pierda parte de su vigencia. Estos reclamos son comprensibles, pero quienes hayan vivido la experiencia de publicar manuscritos en inglés, en revistas con alto prestigio internacional, saben que los pasos recorridos allí varían entre dos semanas (para recibir un rechazo inmediato por el editor, sin 


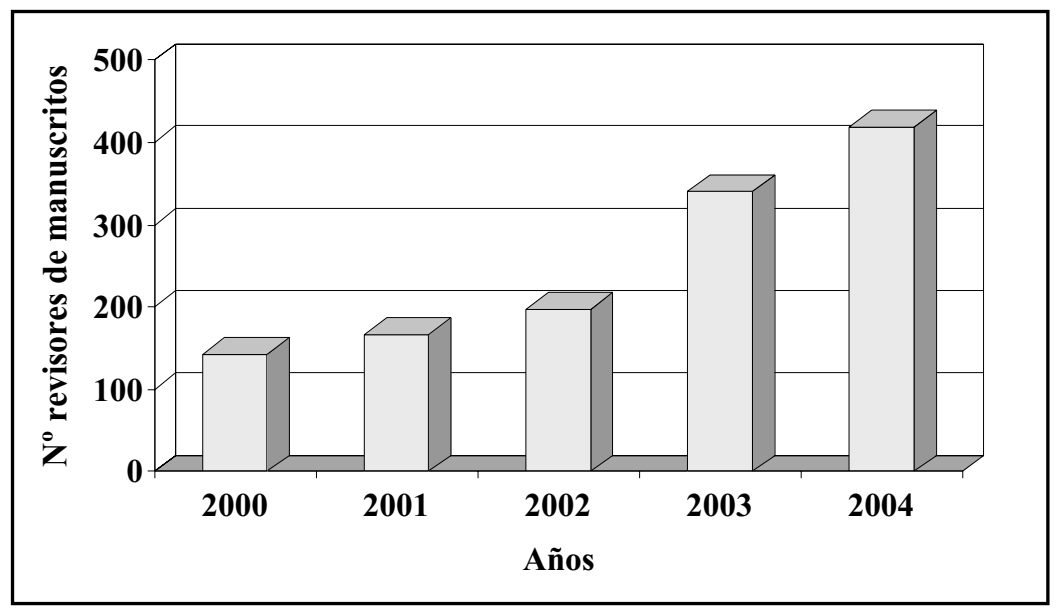

Figura 1. Revisores de manuscritos, años 2000 a 2004, en la Rev Méd Chile

revisión externa del manuscrito) hasta dieciocho meses (para la publicación de una versión final, aceptada por revisores externos y los editores).

Otra consecuencia de la mayor oferta de manuscritos es que en su revisión por pares ha debido participar un número también creciente de revisores (Figura 1). En el año 2004 intervinieron más de 400 personas. Algunas revisaron 20 más manuscritos en el año y los miembros del Comité Editorial Asesor, 3 a 5. Además, los miembros de este Comité asesoran a los editores en la selección de revisores externos, en este proceso altruista, voluntario e indispensable para mejorar la calidad de los manuscritos que se publican ${ }^{5}$. Pero, las múltiples responsabilidades que tenemos los profesionales de la salud y de las ciencias biomédicas, y otras eventualidades, suelen retardar la recepción de sus informes. También suele haber demora en la corrección de los manuscritos por los autores y en la entrega de una segunda versión, que debe ser revisada por los expertos externos y los editores. Se ha solicitado también la opinión de revisores en el extranjero, pero en un número aún pequeño, condicionado a su dominio del idioma del manuscrito (generalmente español), sus lazos con la Revista y la posibilidad de comunicarnos mediante el correo electrónico. El número de manuscritos rechazados definitivamente (sin o con revisión externa) ha aumentado ( $21 \%$ de los manuscritos recibidos, versus $12 \%$ en años previos); su mayor proporción se debió a que los editores consideramos que el tema no correspon- día a los objetivos de esta Revista o los manuscritos tenían deficiencias evidentes.

Las relaciones con los editores de otras revistas y con organizaciones nacionales e internacionales han facilitado el acceso a nuevos conceptos en la edición de revistas biomédicas, en la identificación de problemas que perjudican la calidad de las publicaciones (particularmente en la ética de la investigación biomédica o clínica y en la ética de las publicaciones científicas), el perfeccionamiento del proceso editorial y de la uniformidad que deben cumplir los manuscritos, el análisis crítico de la revisión por pares externos y del rol que deben jugar los editores en el proceso global.

En mayo de 2005 se realizó en Santiago el «I Simposio para Editores de Revistas Científicas Biomédicas Chilenas», organizado por CONICYT y la Asociación Chilena de Editores de Revistas Biomédicas, con el auspicio de la Sociedad Médica de Santiago (Sociedad Chilena de Medicina Interna). Participaron 48 personas (editores de 38 revistas publicadas en Chile, bibliotecólogos y otros profesionales que intervienen en la edición, diagramación, impresión y difusión de estas revistas). El Editor Jefe de la Revista Médica de Chile participó en la organización y coordinación del Simposio y el Dr. Joaquín Palma, Editor Asociado, fue co-director de uno de los talleres de discusión.

En septiembre de 2005 se realizó el \&ifth International Congress on Peer Review and Biomedical Publication», en Chicago, EE UU de NA, organizado por los editores de JAMA y del BMJ 
Publishing Group, con 470 participantes, de revistas publicadas en 38 países y otros profesionales involucrados en la publicación de revistas científicas. El Editor Jefe de la Revista Médica de Chile integró el «Congress Advisory Board»y fue posteriormente designado miembro del $\mathbb{N}$ Nominating Committee» de la World Association of Medical Journal Editors (WAME).

El aumento de la demanda por publicar en revistas médicas es un fenómeno universal, entre cuyas consecuencias está que las revistas con mayor prestigio internacional rechazan 50 a $75 \%$ de los manuscritos que reciben. Todas tratan de perfeccionar su proceso editorial, particularmente reducir el lapso de tiempo que media desde la recepción de un manuscrito hasta que es publicado. Uno de los recursos que utilizan es la aplicación de programas computacionales (software) que permiten ejecutar todas las etapas del proceso editorial en forma semi automática. Las comunicaciones entre los editores, el equipo administrativo, los impresores, los autores y los revisores externos se manejan exclusivamente en la Internet. La confidencialidad de cada etapa se controla mediante claves de acceso individual y con duración limitada. Estos programas han sido incorporados por muchas revistas y su uso es creciente. El costo inicial es alto, exige adaptación para cada revista y supervisión por expertos, aprendizaje por las personas que lo manejan, y ajustes que demoran uno a tres años en perfeccionarse. Pero, finalmente, se cumple el sueño de pasar de la etapa del estafeta («correo ordinario

\section{REFERENCIAS}

1. Reyes H. La Revista Médica de Chile en una biblioteca de ciencias en la Internet (Editorial). Rev Méd Chile 2001; 129: 131-2.

2. Reyes H, Palma J, Andresen M. El manejo editorial de los manuscritos recibidos en la Revista Médica de Chile (Editorial). Rev Méd Chile 2004; 132: 710. que iba a caballo de un lugar a otro», Diccionario de la Real Academia de la Lengua) a la del ciberespacio y cada persona que participa en el proceso (autores, en primer lugar) puede acceder en forma inmediata a información tal como en cuál etapa del proceso se encuentra un manuscrito. ¡No más consultas telefónicas, ni certificados para universidades o entidades que financian la investigación, con datos que se convirtieron en demandas absurdas ya en pleno siglo 20!

Estos programas no están todavía disponibles para revistas que se editan en español. Cuando aparezcan, propondremos su ensayo y eventual aplicación en esta revista. Entretanto, un acuerdo con los impresores permitió aumentar el número de páginas de cada ejemplar de la Revista Médica de Chile y se modificará el diseño de las páginas que muestran información general institucional, para aprovecharlas mejor. Además, mantendremos la política editorial de limitar la extensión de los manuscritos, según su naturaleza y contenidos, para dar espacio a más manuscritos.

Nos pareció apropiado dar esta información sobre la marcha de la Revista. Sería interesante recibir opiniones de los lectores y de los autores de manuscritos, aunque ésta última parecería reflejada en su interés por publicar en ella. La Sección «Cartas al Editor» está disponible para acoger y difundir sus comentarios.

Humberto Reyes B, Editor

Max Andresen H, Joaquín Palma H,

Editores Asociados

3. Crónica. Revisión por pares, año 2003. Rev Méd Chile 2004; 132: 891-5.

4. Crónica. Revisión por pares en la Revista Médica de Chile, año 2004. Rev Méd Chile 2005; 133: 8505.

5. Reyes H, Palma J, Andresen M. El Comité Editorial Asesor: Nuevos miembros con mayores responsabilidades (Editorial). Rev Méd Chile 2005; 133: 910. 